\title{
1 Efficient use of harvest data: An integrated population model for exploited 2 animal populations
}

3

4 Marlène Gamelon ${ }^{1, *}$, Éric Baubet ${ }^{2}$, Aurélien Besnard ${ }^{3}$, Jean-Michel Gaillard ${ }^{4}$, Jean-

5 Dominique Lebreton ${ }^{3}$, Laura Touzot ${ }^{4}$, Lara Veylit $^{1}$, Olivier Gimenez $^{3}$

$7 \quad{ }^{1}$ Centre for Biodiversity Dynamics, Department of Biology, Norwegian University of Science 8 and Technology, 7491 Trondheim, Norway.

$9 \quad{ }^{2}$ Office National de la Chasse et de la Faune Sauvage, 2 Bis Rue des Religieuses, BP 19, 1052120 Châteauvillain, France.

$11{ }^{3}$ Centre d'Écologie Fonctionnelle et Évolutive, Université Montpellier, Université Paul Valéry

12 Montpellier 3, EPHE, IRD, Montpellier, France.

13 Université de Lyon, F-69000, Lyon; Université Lyon 1; CNRS, UMR 5558, Laboratoire de

14 Biométrie et Biologie Évolutive, F-69622, Villeurbanne, France.

16 *E-mail correspondence author: marlene.gamelon@ntnu.no

18 Running headline: Efficient use of harvest data 


\section{Abstract}

1. Many populations are affected by hunting or fishing. Models designed to assess the sustainability of harvest management require accurate estimates of demographic parameters (e.g. survival, reproduction) hardly estimable with limited data collected on exploited populations. The joint analysis of different data sources with integrated population models (IPM) is an optimal framework to obtain reliable estimates for parameters usually difficult to estimate, while accounting for imperfect detection and observation error. The IPM built so far for exploited populations have integrated count-based surveys and catch-at-age data into ageclass structured population models. But the age of harvested individuals is difficult to assess and often not recorded, and population counts are often not performed on a regular basis, limiting their use for the monitoring of exploited populations.

2. Here, we propose an IPM that makes efficient use of data commonly collected in exploited marine and terrestrial populations of vertebrates. As individual measures of body mass at both capture and death are often collected in fish and terrestrial game species, our model integrates capture-mark-recapture-recovery data and data collected at death into a body mass-structured population model. It allows the observed number of individuals harvested to be compared with the expected number and provides accurate estimates of demographic parameters. worldwide, the wild boar Sus scrofa, as a case study. For this species that has increased in distribution and abundance over the last decades, the model provides accurate and precise annual estimates of key demographic parameters (survival, reproduction, growth) and of population size while accounting for imperfect detection and observation error. 
42 4. To avoid an overexploitation of declining populations or an under-exploitation of

43 increasing populations, it is crucial to gain a good understanding of the dynamics of exploited

44 populations. When managers or conservationists have limited demographic data, the IPM

45 offers a powerful framework to assess population dynamics. Being highly flexible, the

46 approach is broadly applicable to both terrestrial and marine exploited populations for which

47 measures of body mass are commonly recorded and more generally, to all populations

48 suffering from anthropogenic mortality causes.

\section{KEYWORDS}

51 body mass-structured population model, exploited populations, harvest, hunting, integrated

52 population model, Sus scrofa 


\section{1 | INTRODUCTION}

Many animal populations are affected by commercial, recreational or subsistence harvest (Lebreton 2005b; Peres 2010; Ripple et al. 2016), i.e. by the removal of individuals through hunting or fishing. Managing such populations to keep the harvest at sustainable levels has long been a central purpose (Williams, Nichols \& Conroy 2002). It is especially true in the current context of global change, as both experimental and observational evidence that harvest may act in synergy with other negative influences such as habitat destruction or disease outcome is accumulating (Camilo et al. 2007; Koons et al. 2015; Chen et al. 2015).

Likewise, in the so-called harvest-interaction hypothesis, harvest might interact with population-level effects of climate change in both marine and terrestrial ecosystems. This interplay between harvest and climate effects may amplify environmentally induced fluctuations in population size and increases extinction risk, or, alternatively, dampen fluctuations and increase population growth rates (Gamelon, Sandercock \& Sæther 2019).

Models designed to assess the sustainability of harvest management typically require accurate estimates of demographic parameters (e.g. survival, reproduction) and population size, which cannot be reliably estimated with limited data. Hence, in particular when demographic information is limited, the challenge is to make efficient use of available data to gain a good understanding of the dynamics of exploited populations and be able, in turn, to provide appropriate management recommendations. When several data types are available, even if each data type by itself provides limited information on demographic parameters, a combined analysis within an integrated population model (IPM) approach offers several advantages (see Schaub \& Abadi 2011; Zipkin \& Saunders 2018 for reviews). First, a combined analysis of different data sources always increases the precision of demographic estimates (for a proof, see Barker \& Kavalieris 2001, and for an example, see table 1 in Péron et al. 2010 who compare parameter estimates and standard errors from a capture-mark- 
recapture only and an integrated-modeling approach). Second, imperfect detection and observation error inherently associated with data sampled in the field (e.g. population counts) are accounted for. Third, the use of IPM allows some parameters that are difficult or impossible to estimate based on separate analyses to be satisfactorily estimated (for an example, see Péron et al. 2010).

From the eighties, the simultaneous analysis of different data sources with IPM has received growing interest in fisheries (see Maunder \& Punt 2013 for a review). Later, IPM have been proposed as powerful tools to assess the dynamics of terrestrial vertebrate populations (e.g. Besbeas et al. 2002 for a study on northern lapwing Vanellus venellus and grey heron Ardea cinerea) and more recently, they have been applied to exploited populations in terrestrial ecosystems (Gauthier et al. 2007; Arnold et al. 2018). Strikingly, IPM built on exploited populations usually integrate population surveys (of alive individuals), catch-at-age data and capture-mark-recapture-recovery (CMRR) data into age-structured population models (Methot Jr \& Wetzel 2013; Arnold et al. 2018; Scheuerell et al. 2019). This state-ofthe-art limited up-to-now the applicability of IPM for two reasons: i) the age of harvested individuals is often not available because its determination is challenging and generally involves expensive and time-demanding analyses. Instead, body mass at capture and/or death are commonly recorded; ii) information on numbers is rarely based on surveys of alive individuals. Instead, the number of individuals removed through exploitation is commonly recorded in many exploited species.

Here, we develop a widely applicable IPM relying on data commonly collected in exploited populations. We illustrate the usefulness of this approach based on the study of a population of an emblematic game species distributed worldwide, the wild boar Sus scrofa. This species has tremendously increased in abundance and distribution over the last decades (Massei et al. 2015), leading to important damage to crops and high risk of disease 
transmission in Europe (see e.g. Schulz et al. 2019). Hunting is commonly assumed to help controlling wild boar expansion. Our model differs from previous IPMs applied to vertebrate populations in two respects: i) typical IPMs integrate data into an age-class structured population model. Instead, we build an IPM that integrates CMRR data and individual measures recorded at death into a body mass-structured population model; ii) both alive and dead individuals are considered in the model allowing us to compare the observed number of individuals shot by hunters in each body mass class to the numbers expected from the IPM. For each mass class, the model allows us to get accurate and precise annual estimates of demographic parameters (i.e. survival, reproduction) and of the number of alive individuals, while accounting for imperfect detection and observation error. Although we targeted wild boar as a case study in this work, the approach we propose can be reliably used for assessing population dynamics of any exploited size-structured population of vertebrates.

\section{2 | MATERIALS AND METHODS}

\section{1 | Demographic data collection}

We studied a wild boar population located in the 11,000 ha forest of ChâteauvillainArc-en-Barrois in North-Eastern France (4802’N, 455’E). Between 1991 and 2016, thanks to an intensive capture-mark-recapture program, 1,152 females were captured from March to September using traps, marked and released in their environment. For each capture event, we recorded the date of capture and body mass. Between October and February, wild boars were harvested. All females shot by hunters (previously marked or not) were weighed and their date of death was accurately recorded. No information was available for individuals that died from natural causes. As wild boar rut generally begins in mid-December, females are often 
pregnant when shot during the hunting season. For pregnant females, the number of fetuses

127 present in the uteri was recorded.

Three types of demographic data were thus available: CMRR data, hunting data and reproduction data.

i) CMRR data provided individual histories of 1,152 marked females together with the body mass at each capture (alive) and when shot (dead). $=4,344$ over the study period).

\section{2 | Population model}

The population vector was considered each year at the end of the hunting season and 
alive individuals), the population vector considered three classes of body mass $(<30 \mathrm{~kg}$ (small), between 30 and $50 \mathrm{~kg}$ (medium) and >50 kg (large) (see Gamelon et al. 2012)) and two states (alive and shot by hunters) for each body mass-class. The population vector had thus 6 components: 3 body mass-classes $\times 2$ states.

The stage-structured matrix population model allows stage-specific population sizes at year $t+1$ to be estimated from stage-specific population sizes at year $t$ and demographic rates (Caswell 2001). We built a female body mass-structured matrix model by considering the three classes of body mass previously defined and the two states: alive and shot by hunters. The output of this body mass-structured matrix model structured according to body mass can be directly compared with the observed distribution of hunted individuals among body massclasses. Females can reproduce in all the three classes. Females may remain in the same body mass-class from one year to the next, with a probability $p S S$ for small females and $p M M$ for medium females. All large females remained in the large body mass class. Alternatively, small females can move to the medium body mass class with a probability $p S M$ or move directly to the largest class with a probability $p S L$ (such as $p S L=1-p S S-p S M$ ). Medium females can move to the next class with a probability $p M L$ (such as 1-pMM). There was no backward transition towards a lighter body mass class. As wild boar females are sedentary (Truvé \& Lemel 2003; Keuling et al. 2010), we assumed no immigration and emigration.

We defined $N_{S, t}$ as the number of small females, $N_{M, t}$ the number of medium females and $N_{L, t}$ the number of large females alive in year $t$. To account for demographic stochasticity in survival processes, we used binomial processes to describe the number of females in each body mass class. 
i. The number of small females alive in year $t N_{S, t}$ is the number of small females in year $t-1$ that remained small plus the number of small females produced by mature females in year $t-1\left(\right.$ NewBorn $\left._{t-1}\right)$ that survived, such as:

$N_{S, t} \sim \operatorname{Bin}\left(N_{S, t-1}, p S S_{t-1} \times S n_{S, t-1} \times\left(1-h_{S, t-1}\right)\right)+\operatorname{Bin}\left(N_{e w B o r n}^{t-1}, S n_{S, t-1} \times(1-h, S, t-1) \times S p n_{t-1} \times p i O s_{t-1}\right)$

where $S n_{j}$ is the survival probability during the first part of the year without hunting, and $1-h_{j}$ probability is defined as $1-N M_{j}$, with $N M_{j}$ the natural mortality bringing together all causes of death except hunting (e.g. diseases). The proportion of females shot by hunters in the class $j h_{j}$ is defined as $\frac{M H_{j}}{1-N M_{j}}$, with $M H_{j}$ the hunting mortality. Assuming that natural mortality was negligible during the hunting period (which is not a very stringent assumption as the multiplicative relationship between natural survival and survival to hunting holds even with some overlap in time between the two sources of mortality), at the end of the year, survival probability for females in the body mass class $j$ equals $S n_{j} \times\left(1-h_{j}\right)=\left(1-N M_{j}\right) \times\left(1-\frac{M H_{j}}{1-N M_{j}}\right)=1-$ year $t-1$ that entered the medium class, plus medium females in year $t-1$ that remained 
iii. The number of large females alive in year $t N_{L, t}$ is the number of small and medium females in year $t-1$ that entered the large class, plus large females in year $t-1$ that survived, such as:

$N_{L, t} \sim \operatorname{Bin}\left(N_{S, t-1}, p S L_{t-1} \times S n_{L, t-1} \times\left(1-h_{L, t-1}\right)\right)+\operatorname{Bin}\left(N_{M, t-1}, p M L_{t-1} \times S n_{L, t-1} \times\left(1-h_{L, t-1}\right)\right)+\operatorname{Bin}\left(N_{L, t-1}\right.$

$\left.S n_{L, t-1} \times\left(1-h_{L, t-1}\right)\right)(4)$.

We used a Poisson distribution for the annual number of newborn females produced by small, medium and large females, such as:

NewBorn $_{t} \sim$ Poisson $\left(N_{S, t} \times B P_{S, t} \times L S_{S, t} \times 0.5+N_{M, t} \times B P_{M, t} \times L S_{M, t} \times 0.5+N_{L, t} \times B P_{L, t} \times L S_{L, t} \times 0.5\right)$

with $B P_{j, t}$ the annual proportion of breeding females and $L S_{j, t}$ the annual litter size of each body mass class $j$. We assumed a balanced sex ratio at birth (Servanty et al. 2007).

In addition to the number of females alive in each body mass class, we estimated the annual body mass-specific numbers of females that were shot $N H_{S, t}, N H_{M, t}$ and $N H_{L, t}$ :

i. The number of small females shot by hunters in year $t N H_{S, t}$ is the number of small females in year $t-1$ that remained small and survived from natural causes but were shot during the hunting season, plus the number of small females produced by mature females in year $t-1$ also shot, such as:

$N H_{S, t} \sim \operatorname{Bin}\left(N_{S, t-1}, p S S_{t-1} \times S n_{S, t-1} \times h_{S, t-1}\right)+\operatorname{Bin}\left(\operatorname{NewBorn}_{t-1}, \operatorname{Sn}_{S, t-1} \times h, S, t-1 \times S p n_{t-1} \times p i O s_{t-1}\right)(6)$.

ii. Similarly, the number of medium females shot by hunters in year $t \mathrm{NH}_{M, t}$ is:

$N H_{M, t} \sim \operatorname{Bin}\left(N_{S, t-1}, p S M_{t-1} \times S n_{M, t-1} \times h_{M, t-1}\right)+\operatorname{Bin}\left(N_{M, t-1}, p M M_{t-1} \times S n_{M, t-1} \times h_{M, t-1}\right)+\operatorname{Bin}$

$\left(\right.$ NewBorn $_{t-1}, S n_{M t-1} \times h_{M, t-1} \times S p n_{t-1} \times\left(1-\right.$ piOs $\left.\left._{t-1}\right)\right)(7)$;

iii. The number of large females shot by hunters in year $t N H_{L, t}$ is: 
$N H_{L, t} \sim \operatorname{Bin}\left(N_{S, t-1}, p S L_{t-1} \times S n_{L, t-1} \times h_{L, t-1}\right)+\operatorname{Bin}\left(N_{M, t-1}, p M L_{t-1} \times S n_{L, t-1} \times h_{L, t-1}\right)+\operatorname{Bin}\left(N_{L, t-1}, S n_{L, t-}\right.$

$\left.215 \quad 1 \times-h_{L, t-1}\right)(8)$.

\section{3 | Model for hunting data}

Hunting data consisted of the annual numbers of females shot by hunters by body

mass classes (small, medium and large), denoted as $y_{S, t}, y_{M, t}$ and $y_{L, t}$, respectively. For these

data, we used the likelihood of a state-space model (de Valpine \& Hastings 2002), which

consists of a process model (i.e. the population model previously described, that is the

population size as a function of demographic rates and the population size the preceding year)

and an observation model. The observation model describes the link between the hunting data

$y_{S, t}, y_{M, t}$ and $y_{L, t}$ and the true number of females shot by hunters in the population $\left(N H_{S, t}, N H_{M, t}\right.$

and $N H_{L, t}$ ) (yellow part, Fig. 1). For each body mass class $j$, we assumed that: $y_{j, t}$

$226 \sim \operatorname{Normal}\left(\mathrm{NH}_{j, t}, \tau_{j, t}\right)$ truncated to positive values, with $\tau_{j, t}=\frac{1}{\sigma_{j}^{2} \times y_{j, t}^{2}}$, where $\sigma_{j}^{2}$ is the term we are

estimating. $\tau_{j, t}$ is the observation error and incorporates both errors in hunting data and lack of

fit of the state equations to the true dynamics of the population (see p. 230 in Schaub \& Abadi

2011). Note the trick often used in JAGS that consists in defining the distributions by their

230 precision $\tau_{j, t}$ rather than their variance $\sigma_{j}^{2}$, the precision being the inverse of the variance. The

likelihood of the state-space model is then the product of the likelihood of the process and the 


\section{4 | Model for capture-mark-recapture-recovery data}

CMRR data were analysed using a multistate model (see Lebreton et al. 2009 for a review) that allows natural mortality $N M$ to be estimated separately from hunting mortality MH (Lebreton, Almeras \& Pradel 1999; Gamelon et al. 2012) for each body mass class (grey part, Fig. 1). We described the fate of an individual using ten states (see Appendix S1). States

1, 2 and 3 were for individuals alive in the small, medium and large body mass classes, causes, again in the three body mass classes, respectively. The state "dead from a natural cause" cannot be observed because no information was available for individuals that did not die from hunting. State 10 corresponded to individuals already dead. The state "already dead" cannot be observed either but brought together all the dead individuals. To account for the dependency between states (i.e. from year $t$ to $t+1$, individuals either survive, die shot by hunters or die from natural causes, see Appendix S1), we used a Dirichlet distribution to model the transitions between states thus ensuring that the sum of these probabilities always equals to 1 . The parameters in the multistate model were annual natural mortality $N M_{j, t}$ and hunting mortality $M H_{j, t}$ for each body mass class $j$. Moreover, yearly transition probabilities from one body mass class to the next (i.e. $p S S_{t}, p S M_{t}, p S L_{t}, p M M_{t}, p M L_{t}$ ) were estimated. To account for the dependency between transition probabilities (i.e. $p S L_{t}=1-p S S_{t}-p S M_{t}$ ), we used a Dirichlet distribution to model the transitions from the small body mass class to the others. This ensures that the sum of the probabilities (i.e. $p S S_{t}+p S M_{t}+p S L_{t}$ ) always equals to 1. Regarding the observation process, if an individual was alive, it could be recaptured with

257 probability $p$ or not recaptured with probability $1-p$; if an individual just died from hunting, its death could be reported "dead recovery" with probability $r$ or not reported with probability 1 - 
$r$. Recapture and recovery probabilities were also time and body mass-class dependent (see Appendix S2 and S3).

\section{5 | Model for reproduction data}

Reproduction data consisted of the annual numbers of pregnant females shot by body mass classes (small, medium and large), denoted as $R_{S, t}, R_{M, t}$ and $R_{L, t}$, respectively and the annual numbers of fetuses counted by body mass classes, denoted as $J_{S, t}, J_{M, t}$ and $J_{L, t}$, respectively. We assumed that the number of fetuses per body mass class $J_{j, t}$ is Poisson distributed such as: $J_{j, t} \sim$ Poisson $\left(R_{j, t} \times L S_{j, t}\right)$, where $L S_{j, t}$ is the term we are estimating, that is the litter size for a female belonging to the body mass class $j$ (blue part, Fig. 1).

\section{6 | Model implementation}

Assuming independence among the datasets, the likelihood of the IPM was the product of the likelihoods of the three different datasets (Besbeas et al. 2002; Kéry \& Schaub 2012): hunting data, CMRR data and reproduction data. The IPM was fit within the Bayesian framework and we used non-informative priors for all the parameters. We only constrained $\sigma_{j}$ in the state-space model to be small using a Uniform distribution between 0 and 0.1 , so that the observation errors $\tau_{j, t}=\frac{1}{\sigma_{j}^{2} \times y_{j, t}^{2}}$ can be large. Markov chain Monte Carlo (MCMC) simulation was used for parameter estimation. All the parameters described in Fig. 1 were estimated within the IPM, even parameters difficult to estimate and often assessed from expert opinion such as Spn and piOs (see Gamelon et al. 2012). The analyses were implemented using JAGS (Plummer 2003) version 4.3.0 called from R version 3.4.3 (R 
281 Development Core Team 2017) with package rjags (Plummer 2016). The JAGS code for

282 fitting the IPM is available in Appendix S3.

283 To assess convergence, we ran three independent chains for 230,000 MCMC

284 iterations, with an adaptation of 180,000 iterations thinning every 100th observation resulting

285 in 1,500 posterior samples. We used the Brooks and Gelman diagnostic $R \square$ to assess the

286 convergence of the simulations and used the rule $R^{\square}<1.2$ to determine whether convergence

287 has been reached (Brooks \& Gelman 1998). Convergence was reached for most of the nodes

288 except for four years (1996-1999) in which not all parameters had converged. To ensure that

289 the priors for initial population sizes do not influence estimates of demographic rates and

290 body mass-specific numbers the first year of the study (i.e., in 1991), only the years between

2911992 and 2016 were included in the analyses.

3 | RESULTS

\subsection{Recapture, recovery and mortality probabilities} recapture probabilities over years are consistent with earlier studies (see Appendix $\mathrm{C}$ in

299 Servanty et al. 2010). In accordance with previous work (Toïgo et al. 2008; Servanty et al. 2010; Gamelon et al. 2011), dead recovery reporting probabilities were high for all body mass classes, ranging from 0.75 to 0.97 for small females (mean $r_{S}=0.90$ ), from 0.79 to 0.96 for females in the medium body mass class (mean $r_{M}=0.88$ ) and from 0.74 to 0.93 for large females (mean $r_{L}=0.84$ ). Mortality probability also fluctuated over the study period (Fig. 2

304 A,B). As expected for this heavily hunted population, hunting mortality $M H$ was much higher 
305

306

307

308

309

310

311

312

313

314

315

316

317

318

319

320

321

322

323

324

325

326

327

than natural mortality $N M$ for small and medium females. Interestingly, the estimate of the mean post-natal survival was 0.60 [ $\min =0.22 ; \max =0.88]$ over the study period, and thus close to the value generally set by expert opinion (0.75, see e.g. Gamelon et al. 2012) .

\subsection{Transition probabilities among body-mass classes}

Females in the small body mass class had an estimated probability of 0.48 to remain in this class on the average ( $p S S$ ) (Fig. 2C). Alternatively, they moved to the medium-sized class with an estimated probability $p S M$ equal to 0.19 on the average, and to the large-sized class with a probability $p S L$ of 0.33 . Females in the medium body mass class had an estimated probability of 0.41 to enter the large class on the average $(p M L)$, other medium females remaining in the medium-sized class $(p M M)$. Newborn females had an estimated probability of 0.88 [ $\min =0.56 ; \max =0.98]$ to remain in the small body mass class (piOs) on the average, this probability being usually set to 0.60 by expert opinion (see e.g. Gamelon et al. 2012).

\section{3 $\mid$ Reproduction}

For reproduction parameters, litter sizes $L S$ were strongly body mass-specific, being the largest for large females (mean $L S_{L}=6.08$ young) and the smallest for females of the smallsized class (mean $L S_{S}=4.21$ young) (Fig. 3). These results are in accordance with previous studies (Gamelon et al. 2013). As for mortality probabilities, litter sizes fluctuated a lot over the study period, from 1.79 to 7.69 young produced for small females $\left(L S_{S}\right)$, from 3.91 to 7.50 young for females in the medium weight class $\left(L S_{M}\right)$ and from 4.97 to 7 young for large females $\left(L S_{L}\right)$. Breeding proportions, i.e. the proportion of breeding females among those in each body mass class, fluctuated over years, especially for females in the small body mass 
class. These probabilities ranged from 0.36 to 0.84 (mean $B P_{S}=0.58$ ) for small females, from

0.43 to 0.65 (mean $B P_{M}=0.53$ ) for medium females, and from 0.48 to 0.61 (mean $B P_{L}=0.53$ ) for large females.

\section{4 | Population sizes}

High annual fluctuations in mortality, transition between body mass classes and reproductive rates translated to high variation in population size over years (Fig. 4). Each year, with a mean of $\mathrm{NH}_{S}=143$ females, most of the females removed from hunting belonged to the small-sized class (Fig. 4A). The mean number of females shot by hunters in the medium class $N H_{M}$ was estimated to be 81 , whereas they were very few large females in the hunting bags (mean $N H_{L}=35$ ). Noticeably, the numbers of females shot in each body mass class expected from the IPM was very close to the observed numbers $y_{j}$ (dots in Fig. 4A). Observation errors $\sigma$ were estimated at 0.096 for small and medium females and 0.099 for large females. In terms of numbers of females alive in the population, small females constituted the largest part of the population (mean number of small females $N_{S}=290$ ), followed by medium (mean $N_{M}=77$ ) and then large females (mean $N_{L}=36$ ) (Fig. 4B).

\section{4 | DISCUSSION}

We develop here an IPM that makes efficient use of data commonly collected in exploited populations, i.e. body mass at captures/death as well as number of individuals removed by harvesting. Our model makes it possible to directly compare the observed and the expected number of individuals shot. It also provides accurate and precise estimates of key demographic parameters, including some that cannot be estimated from separate analyses. 
351 Using a wild boar population as a case study, we demonstrate that this framework is a

352 powerful tool to gain a good understanding of the dynamics of exploited populations.

\subsection{A comprehensive picture of population dynamics}

In accordance with previous work (Toïgo et al. 2008; Gamelon et al. 2011), we

showed that hunting mortality probabilities $M H$ are high for all body mass classes.

357 Conversely, natural mortality $N M$ was low (Fig. 2 A,B). This is expected among ungulates

where the average natural adult survival probability $S n$ often exceed 0.95 in females (Gaillard

et al. 2000). These hunting and natural mortality patterns are in accordance with other hunted

wild boar populations (see Gamelon 2019 for a review). Our IPM thus confirms that hunting

is an important driver of wild boar population dynamics. Regarding to reproduction

parameters, wild boar is a highly fecund species, being able to produce large litters (Fig. 3) as

early as their first year of life (Servanty et al. 2009) at a body mass lower than $30 \mathrm{~kg}$ (small

mass-class). Together with reduced survival due to hunting, this leads to a short generation

time, i.e. a low mean age of mothers at childbirth (Gaillard et al. 2005, 2016) close to two

years, whereas it is close to six years for similar-sized ungulates (Servanty et al. 2011). This

367

unusual life history among ungulates (Focardi et al. 2008), characterized with a fast turnover

of individuals in the population, explains why the number of individuals in the population did

not collapse (Fig. 4B) during the study period despite such a high hunting pressure, and why 


\subsection{Estimates for demographic parameters hardly measurable in the field} alive, which are usually needed to make relevant management recommendations.

Interestingly, the model also allows us to estimate some growth parameters that are difficult to measure in the field, because they would require multiple captures of the same individuals

Baubet et al. (2009) aimed to tag piglets inside their birth nest to assess post-natal survival

locate the birth nests, but also because it induced abandonment of the piglets after tagging. By

jointly analysing different data sources, the IPM is a powerful tool to achieve such a goal.

\subsection{A framework based on data commonly collected in exploited populations}


boar populations, but it is clearly applicable to many other populations in both terrestrial and marine environments. For instance, a lot of commercially important marine fish species are subject to strong harvesting (Pauly et al. 2002; Hutchings \& Reynolds 2004). The IPM can be a suitable tool to anticipate the collapse of some of them (Hutchings \& Myers 1994; Myers, Hutchings \& Barrowman 1997) by making efficient use of the limited data available (see Maunder 2004; Saunders, Cuthbert \& Zipkin 2018 for the use of IPM in prospective analyses).

The use of IPM as a management tool is not novel in fisheries (see Maunder \& Punt 2013 for a review) but they are to date based on age-structured population models. However, fish are indeterminate growers for which demographic parameters are usually strongly massdependent. Moreover, while taking reliable mass measurements of animals killed by humans is straightforward, information on age is more cumbersome and often inaccurate and thus recorded in very few populations only. Even in determinate growers such as birds and mammals, evidence is accumulating that body mass is a crucial structuring factor of population dynamics (Gaillard et al. 2000 for a review on large herbivores; see Coulson, Tuljapurkar \& Childs 2010 for a case study on Soay sheep Ovis aries), making biologically relevant the use of body mass-structured models in IPM for a large range of exploited populations of vertebrates. More generally, our framework based on a body mass-structured model adds to the spate of studies that have recently flourished in the literature showing that trait-based approaches (such as body mass) and demographic approaches are intertwined (Salguero-Gómez et al. 2018; Smallegange \& Berg 2019). Another clear advantage of our IPM is the inclusion of the number of dead individuals in the body mass-structured population model, whereas all the IPMs built so far only included the number of individuals alive in a population model (Caswell 2001). In exploited populations, getting information on the number of individuals alive is challenging whereas information on the number of individuals 
424 killed (by hunting or fishing) is often available. This number is even almost perfectly known

425 in our population. With the inclusion of information related to hunting bags, our IPM renders

426 possible a direct comparison between observed and predicted numbers of individuals shot in

427 each body-mass class, a crucial demographic information in exploited populations.

The use of the number of individuals harvested in our IPM could be modified to the

Fleming 2018). Such estimates of age-and sex structures among dead individuals have been

used up to now in an ad hoc fashion to match model predictions (Holopainen et al. 2018). The

analysis of such data based on an IPM would certainly greatly improve our knowledge and between sexes in duck populations. wandering albatross Diomedea exulans). Beyond mortality due to hunting and fishing, the

444 IPM can also be expanded to all forms of anthropogenic mortalities (see e.g. Chevallier et al. 
447 IPM, it is straightforward to integrate a priori biological knowledge by setting some

448 parameters to known values or by using informative priors.

\section{Conclusions}

454 is flexible and can be adapted to the life history and the data available for the population of

455 interest. This model that integrates commonly collected data in marine and terrestrial

456 exploited populations is therefore widely applicable.

ACKNOWLEDGMENTS

459 Wild boar data collection was performed and granted by the French National Agency for

460 Wildlife and Hunting (ONCFS). We are grateful to all those who helped marking and

461 collecting harvested wild boar, in particular Serge Brandt, Eveline Nivois and Cyril Rousset,

462 to the Office National des Forêts and to F. Jehlé who allowed us to work on the study area.

463 This work was supported by the Research Council of Norway through its Centre of

464 Excellence funding scheme, Project Number 223257. 


\section{AUTHOR'S CONTRIBUTIONS}

467 EB contributed to data collection. MG, EB, AB, JMG, JDL and OG conceived the study. MG

468 conducted the analyses with insights from OG, wrote the first draft and all authors contributed

469 to revisions of the initial manuscript.

The data supporting the results will be archived in an appropriate public repository in Dryad,

473 should the manuscript be accepted.

\section{REFERENCES}

476

477
Arnold, T.W., Clark, R.G., Koons, D.N. \& Schaub, M. (2018) Integrated population models facilitate ecological understanding and improved management decisions. The Journal of Wildlife Management, 82, 266-274.

Barker, R.J. \& Kavalieris, L. (2001) Efficiency gain from auxiliary data requiring additional nuisance parameters. Biometrics, 57, 563-566.

Baubet, E., Servanty, S. \& Brandt, S. (2009) Tagging piglets at the farrowing nest in the wild: some preliminary guidelines. Acta Silvatica et Lignaria Hungarica, 5, 159-166.

Besbeas, P., Freeman, S.N., Morgan, B.J.T. \& Catchpole, E.A. (2002) Integrating markrecapture-recovery and census data to estimate animal abundance and demographic parameters. Biometrics, 58, 540-547.

Bonenfant, C., Gaillard, J., Coulson, T., Festa $\square$ Bianchet, M., Loison, A., Garel, M., Loe, L.E., Blanchard, P., Pettorelli, N., Owen $\square$ Smith, N., Du Toit, J. \& Duncan, P. (2009) Empirical evidence of density $\square$ dependence in populations of large herbivores. Advances in Ecological Research (ed H. Caswell), pp. 313-357. Academic Press.

Brooks, S.P. \& Gelman, A. (1998) General methods for monitoring convergence of iterative simulations. Journal of Computational and Graphical Statistics, 7, 434-455.

Camilo, C., Metzger, R., Rollo, A. \& Myers, R.A. (2007) Experimental simulations about the effects of overexploitation and habitat fragmentation on populations facing 
environmental warming. Proceedings of the Royal Society B: Biological Sciences, 274, 1023-1028.

Caswell, H. (2001) Matrix Population Models: Construction, Analysis, and Interpretation. Sinauer Associates.

Chen, S., Fan, Z., Roby, D.D., Lu, Y., Chen, C., Huang, Q., Cheng, L. \& Zhu, J. (2015) Human harvest, climate change and their synergistic effects drove the Chinese Crested Tern to the brink of extinction. Global Ecology and Conservation, 4, 137-145.

Chevallier, C., Hernández $\square$ Matías, A., Real, J., Vincent $\square$ Martin, N., Ravayrol, A. \& Besnard, A. (2015) Retrofitting of power lines effectively reduces mortality by electrocution in large birds: an example with the endangered Bonelli's eagle. Journal of Applied Ecology, 52, 1465-1473.

Coulson, T., Tuljapurkar, S. \& Childs, D.Z. (2010) Using evolutionary demography to link life history theory, quantitative genetics and population ecology. Journal of Animal Ecology, 79, 1226-1240.

Focardi, S., Gaillard, J.-M., Ronchi, F. \& Rossi, S. (2008) Survival of wild boars in a variable environment: unexpected life-history variation in an unusual ungulate. Journal of Mammalogy, 89, 1113-1123.

Gaillard, J.-M., Festa-Bianchet, M., Yoccoz, N.G., Loison, A. \& Toïgo, C. (2000) Temporal variation in fitness components and population dynamics of large herbivores. Annual Review of Ecology and Systematics, 31, 367-393.

Gaillard, J.-M., Lemaître, J.-F., Berger, V., Bonenfant, C., Devillard, S., Douhard, M., Gamelon, M., Plard, F. \& Lebreton, J.-D. (2016) Axes of variation in life histories. Encyclopedia of Evolutionary Biology, Elsevier, pp. 312-323. R. M. Kliman, Oxford, UK.

Gaillard, J.-M., Yoccoz, N.G., Lebreton, J.-D., Bonenfant, C., Devillard, S., Loison, A., Pontier, D. \& Allaine, D. (2005) Generation time: a reliable metric to measure lifehistory variation among mammalian populations. The American Naturalist, 166, 119123.

Gamelon, M. (2019) Hunting, predation and senescence in boars. Reference Module in Biomedical Sciences

Gamelon, M., Besnard, A., Gaillard, J.-M., Servanty, S., Baubet, E., Brandt, S. \& Gimenez, O. (2011) High hunting pressure selects for earlier birth date: wild boar as a case study. Evolution, 65, 3100-3112.

Gamelon, M., Gaillard, J.-M., Baubet, E., Devillard, S., Say, L., Brandt, S. \& Gimenez, O. (2013) The relationship between phenotypic variation among offspring and mother body mass in wild boar: evidence of coin-flipping? Journal of Animal Ecology, 82, 937-945.

Gamelon, M., Gaillard, J.-M., Servanty, S., Gimenez, O., Toïgo, C., Baubet, E., Klein, F. \& Lebreton, J.-D. (2012) Making use of harvest information to examine alternative 
management scenarios: a body weight-structured model for wild boar. Journal of Applied Ecology, 49, 833-841.

Gamelon, M., Sandercock, B.K. \& Sæther, B.-E. (2019) Does harvesting amplify environmentally induced population fluctuations over time in marine and terrestrial species? Journal of Applied Ecology, 56, 2186-2194.

Gauthier, G., Besbeas, P., Lebreton, J.-D. \& Morgan, B.J.T. (2007) Population growth in snow geese: a modeling approach integrating demographic and survey information. Ecology, 88, 1420-1429.

Holopainen, S., Christensen, T.K., Pöysä, H., Väänänen, V.-M., Rintala, J. \& Fox, A.D. (2018) Associations between duck harvest, hunting wing ratios and measures of reproductive output in Northern Europe. European Journal of Wildlife Research, 64, 72.

Hutchings, J.A. \& Myers, R.A. (1994) What can be learned from the collapse of a renewable resource? Atlantic cod, Gadus morhua, of Newfoundland and Labrador. Canadian Journal of Fisheries and Aquatic Sciences, 51, 2126-2146.

Hutchings, J.A. \& Reynolds, J.D. (2004) Marine Fish Population Collapses: Consequences for Recovery and Extinction Risk. BioScience, 54, 297-309.

Kéry, M. \& Schaub, M. (2012) Bayesian Population Analysis using WinBUGS: A hierarchical perspective. Academic Press, Boston.

Keuling, O., Lauterbach, K., Stier, N. \& Roth, M. (2010) Hunter feedback of individually marked wild boar Sus scrofa L.: dispersal and efficiency of hunting in northeastern Germany. European Journal of Wildlife Research, 56, 159-167.

Koons, D.N., Colchero, F., Hersey, K. \& Gimenez, O. (2015) Disentangling the effects of climate, density dependence, and harvest on an iconic large herbivore's population dynamics. Ecological Applications, 25, 956-967.

Lebreton, J.-D. (2005a) Age, stages, and the role of generation time in matrix models. Ecological Modelling, 188, 22-29.

Lebreton, J.-D. (2005b) Dynamical and statistical models for exploited populations. Australian \& New Zealand Journal of Statistics, 47, 49-63.

Lebreton, J.-D., Almeras, T. \& Pradel, R. (1999) Competing events, mixtures of information and multistratum recapture models. Bird Study, 46, S39-46.

Lebreton, J., Nichols, J.D., Barker, R.J., Pradel, R. \& Spendelow, J.A. (2009) Modeling individual animal histories with multistate capture-recapture models. Advances in Ecological Research, 41, 87-173.

Massei, G., Kindberg, J., Licoppe, A., Gačić, D., Šprem, N., Kamler, J., Baubet, E., Hohmann, U., Monaco, A., Ozoliņ̌s, J., Cellina, S., Podgórski, T., Fonseca, C., Markov, N., Pokorny, B., Rosell, C. \& Náhlik, A. (2015) Wild boar populations up, numbers of hunters down? A review of trends and implications for Europe. Pest Management Science, 71, 492-500. 
Maunder, M.N. (2004) Population viability analysis based on combining Bayesian, integrated, and hierarchical analyses. Acta Oecologica, 26, 85-94.

Maunder, M.N. \& Punt, A.E. (2013) A review of integrated analysis in fisheries stock assessment. Fisheries Research, 142, 61-74.

Methot Jr, R.D. \& Wetzel, C.R. (2013) Stock synthesis: a biological and statistical framework for fish stock assessment and fishery management. Fisheries Research, 142, 86-99.

Myers, R.A., Hutchings, J.A. \& Barrowman, N.J. (1997) Why do fish stocks collapse? The example of cod in Atlantic Canada. Ecological Applications, 7, 91-106.

Pardo, D., Barbraud, C. \& Weimerskirch, H. (2014) What shall I do now? State-dependent variations of life-history traits with aging in Wandering Albatrosses. Ecology and Evolution, 4, 474-487.

Pauly, D., Christensen, V., Guénette, S., Pitcher, T.J., Sumaila, U.R., Walters, C.J., Watson, R. \& Zeller, D. (2002) Towards sustainability in world fisheries. Nature, 418, 689.

Peres, C.A. (2010) Overexploitation. Conservation Biology for all, pp. 107-130. Oxford University Press, Oxford.

Péron, G., Crochet, P.-A., Doherty, P.F. \& Lebreton, J.-D. (2010) Studying dispersal at the landscape scale: efficient combination of population surveys and capture-recapture data. Ecology, 91, 3365-3375.

Plummer, M. (2003) JAGS: A Program for Analysis of Bayesian Graphical Models Using Gibbs Sampling. Proceedings of the 3rd International Workshop on Distributed Statistical Computing, pp. 20-22. Hornik K, Leisch F, Zeileis A, Vienna, Austria.

Plummer, M. (2016) rjags: Bayesian graphical models using MCMC.

R Development Core Team. (2017) R: A language and environment for statistical computing.

Raftovich, R.V., Chandler, S.C. \& Fleming, K.K. (2018) Migratory bird hunting activity and harvest during the 2016-17 and 2017-18 hunting seasons.

Ripple, W.J., Abernethy, K., Betts, M.G., Chapron, G., Dirzo, R., Galetti, M., Levi, T., Lindsey, P.A., Macdonald, D.W., Machovina, B., Newsome, T.M., Peres, C.A., Wallach, A.D., Wolf, C. \& Young, H. (2016) Bushmeat hunting and extinction risk to the world's mammals. Royal Society Open Science, 3, 160498.

Salguero $\square$ Gómez, R., Violle, C., Gimenez, O. \& Childs, D. (2018) Delivering the promises of trait-based approaches to the needs of demographic approaches, and vice versa. Functional Ecology, 32, 1424-1435.

Saunders, S.P., Cuthbert, F.J. \& Zipkin, E.F. (2018) Evaluating population viability and efficacy of conservation management using integrated population models. Journal of Applied Ecology, 55, 1380-1392.

Schaub, M. \& Abadi, F. (2011) Integrated population models: a novel analysis framework for deeper insights into population dynamics. Journal of Ornithology, 152, 227-237. 
Scheuerell, M.D., Ruff, C.P., Anderson, J.H. \& Beamer, E.M. (2019) An integrated population model for estimating the relative effects of natural and anthropogenic factors on a threatened population of Pacific trout. bioRxiv, 734996.

Schulz, K., Oḷ̌̌evskis, E., Staubach, C., Lamberga, K., Seržants, M., Cvetkova, S., Conraths, F.J. \& Sauter-Louis, C. (2019) Epidemiological evaluation of Latvian control measures for African swine fever in wild boar on the basis of surveillance data. Scientific Reports, 9, 4189.

Servanty, S., Choquet, R., Baubet, E., Brandt, S., Gaillard, J.-M., Schaub, M., Toïgo, C., Lebreton, J.-D., Buoro, M. \& Gimenez, O. (2010) Assessing whether mortality is additive using marked animals: a Bayesian state-space modeling approach. Ecology, 91, 1916-1923.

Servanty, S., Gaillard, J.-M., Toïgo, C., Brandt, S. \& Baubet, E. (2009) Pulsed resources and climate-induced variation in the reproductive traits of wild boar under high hunting pressure. Journal of Animal Ecology, 78, 1278-1290.

Smallegange, I.M. \& Berg, M.P. (2019) A functional trait approach to identifying life history patterns in stochastic environments. Ecology and Evolution, 9, 9350-9361.

Toïgo, C., Servanty, S., Gaillard, J.-M., Brandt, S. \& Baubet, E. (2008) Disentangling natural from hunting mortality in an intensively hunted wild boar population. The Journal of Wildlife Management, 72, 1532-1539.

Truvé, J. \& Lemel, J. (2003) Timing and distance of natal dispersal for wild boar Sus scrofa in Sweden. Wildlife Biology, 9, 51-57.

de Valpine, P. \& Hastings, A. (2002) Fitting population models incorporating process noise and observation error. Ecological Monographs, 72, 57-76.

Williams, B.K., Nichols, J.D. \& Conroy, M.J. (2002) Analysis and Management of Animal Populations. Academic Press, San Diego.

Zipkin, E.F. \& Saunders, S.P. (2018) Synthesizing multiple data types for biological conservation using integrated population models. Biological Conservation, 217, 240 250 . 
Figure 1. Directed acyclic graph of the IPM. Squares represent the data, circles represent the parameters to be estimated. Arrows represent dependencies. Three types of data are collected: CMRR data, hunting data $\left(y_{j}\right)$ and reproductive data (number of pregnant females $(R)$ and number of fetuses $(J)$ in each body mass class $j$ ). Estimated parameters are the body mass-specific recapture probability $p_{j}$, recovery probability $r_{j}$, natural mortality $N M_{j}$, hunting mortality $M H_{j}$, litter size $L S_{j}$, numbers of females alive $N_{i}$, numbers of females shot by hunters $N H_{j}$, breeding proportions $B P_{j}$, post-natal survival $S p n$ (from birth to weaning), observation error $\sigma_{j}^{2}$ and transitions between body mass classes (growth) with piOs the probability for newborn remaining in the small body mass class, $p S S$ the probability of small females remaining in this class during the year, $p S M$ the probability of small females entering the medium class, $p S L$ the probability of small females entering the large class, $p M M$ the probability of medium females remaining in this class during the year and $p M L$ the probability of medium females entering the large class. 


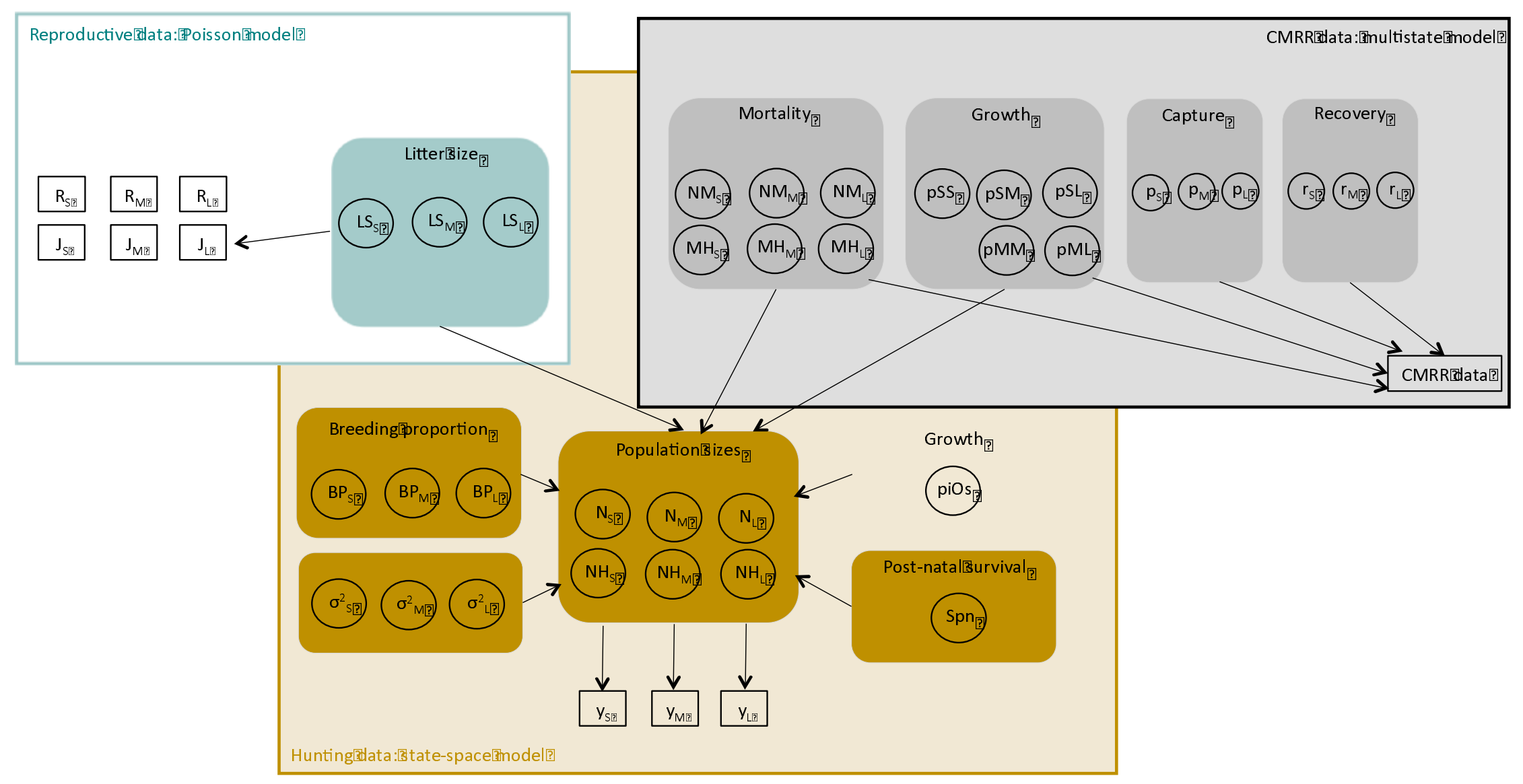


Figure 2. Mortality and growth parameters estimated with the IPM (grey part in Fig. 1). Posterior means of A) annual hunting mortality $M H$ and B) annual natural mortality $N M$ for each body mass class (small S, medium M and large L). Posterior means of C) transition probability between body mass classes ( $p S S$ : probability of small females remaining in this class; $p S M$ : probability of small females entering the medium class; $p S L$ : probability of small females entering the large class; $p M L$ : probability of medium females entering the large class during the year) for the wild boar population at Châteauvillain-Arc-en-Barrois between 1992 and 2015. CRI are not shown to improve the readability. 
A)

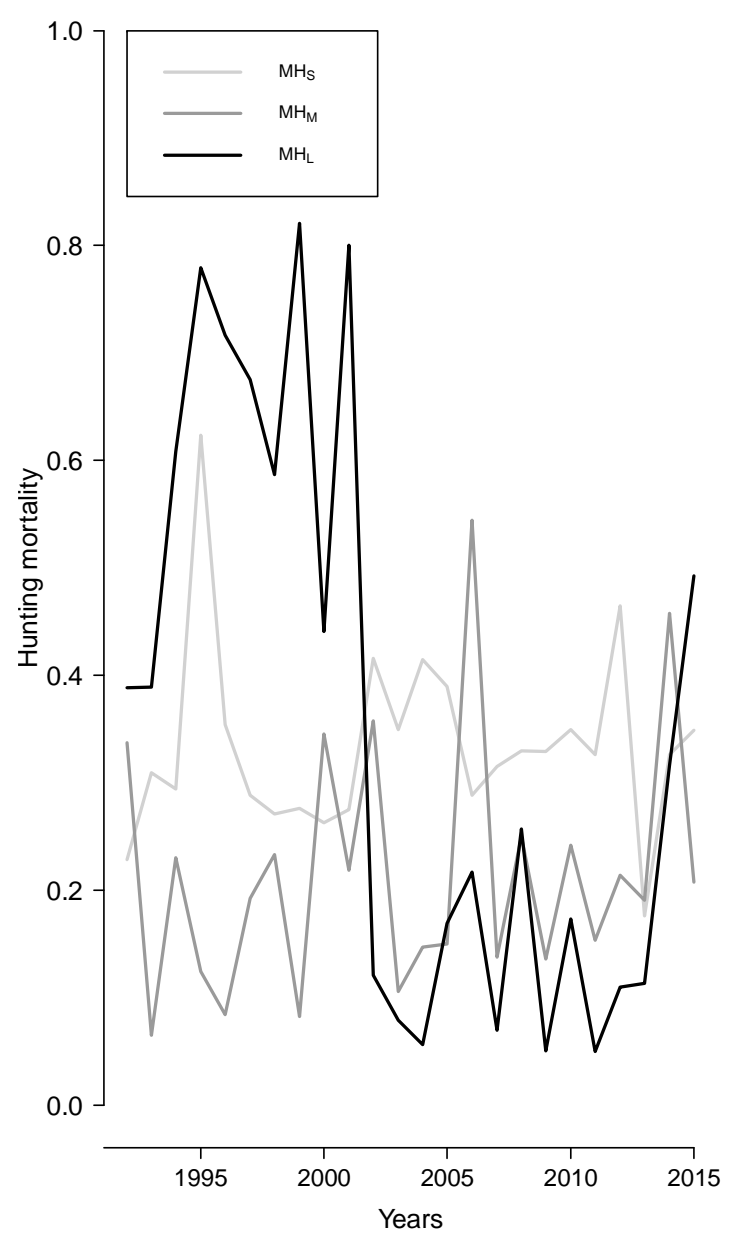

B)

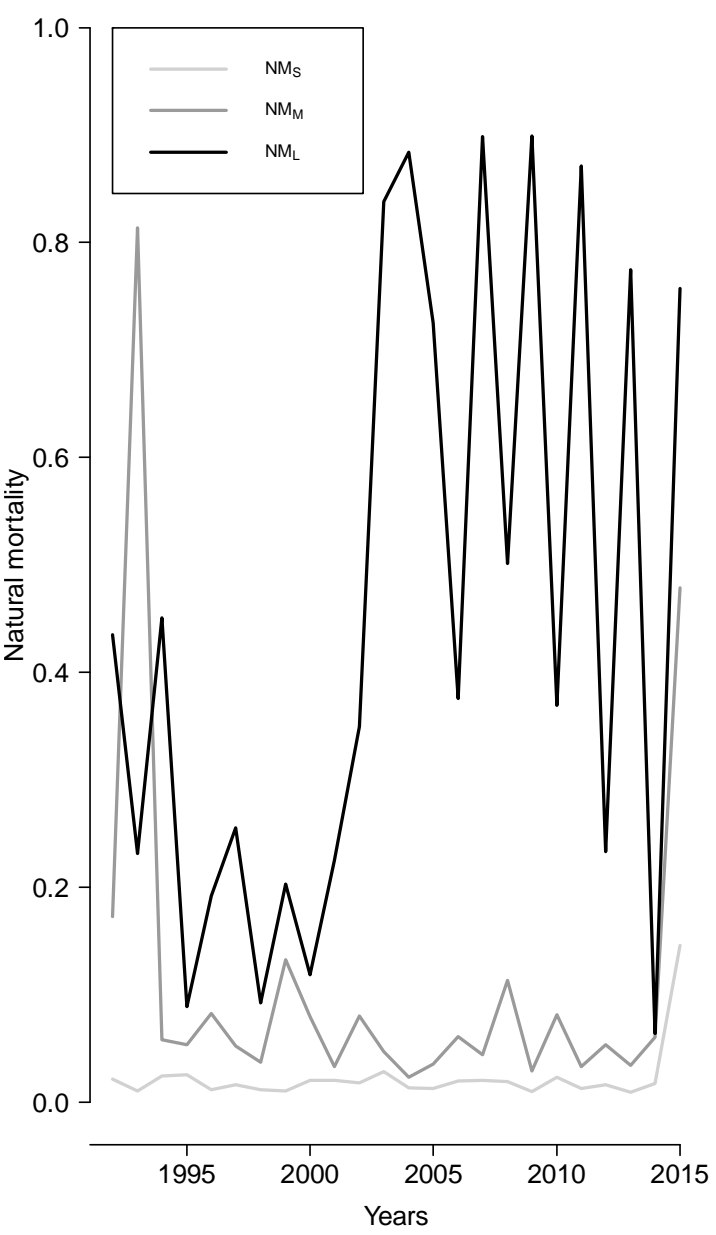

C)

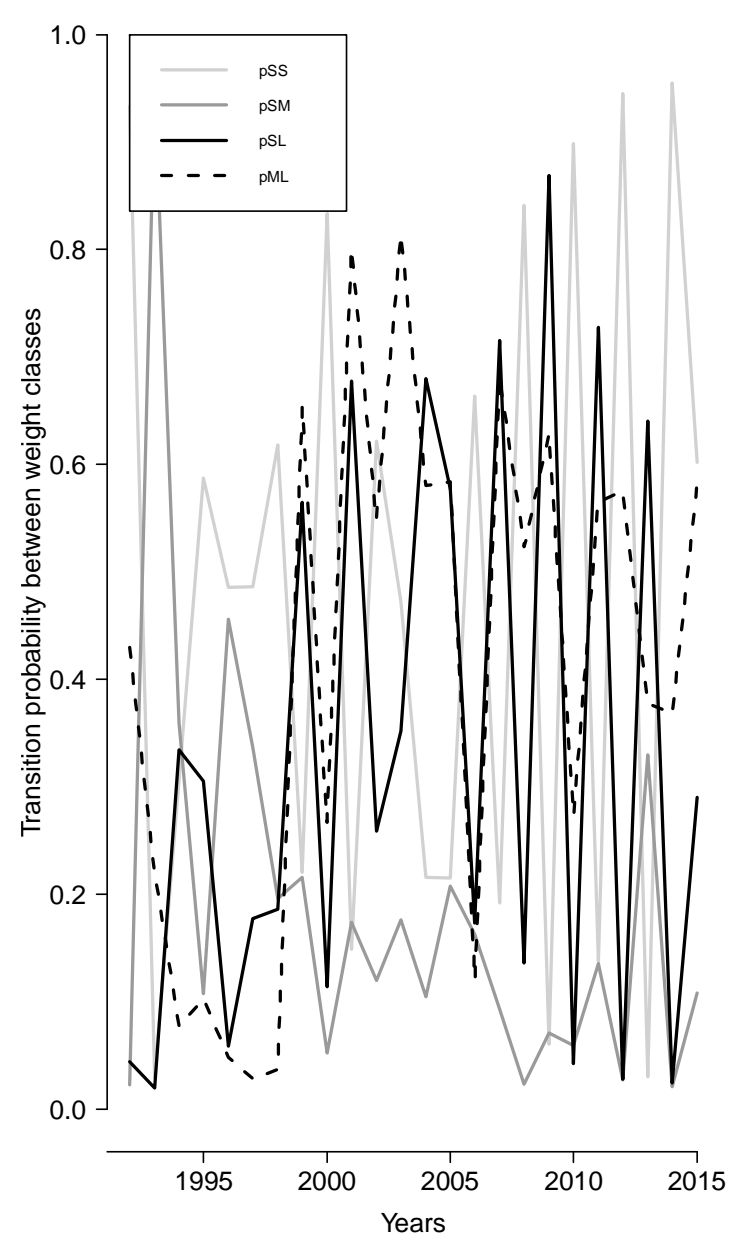









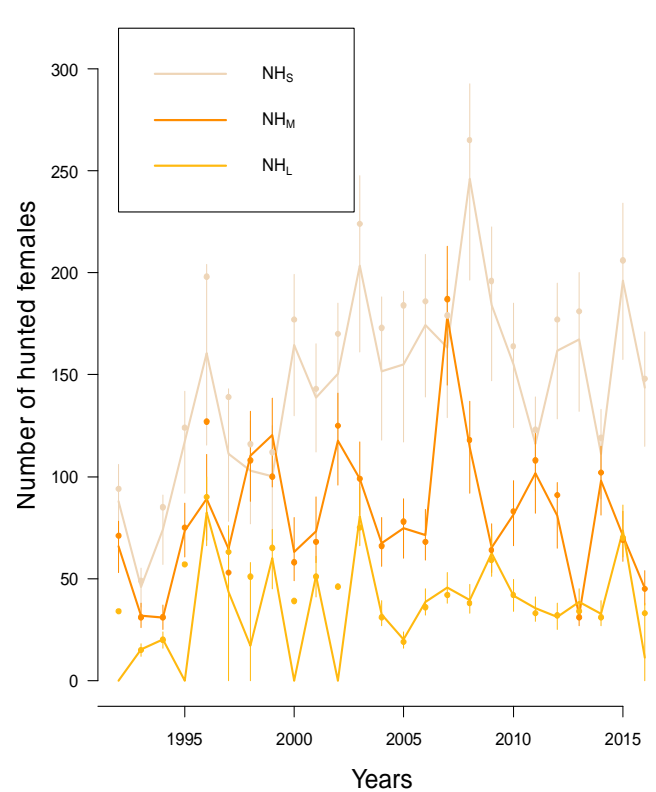

B)

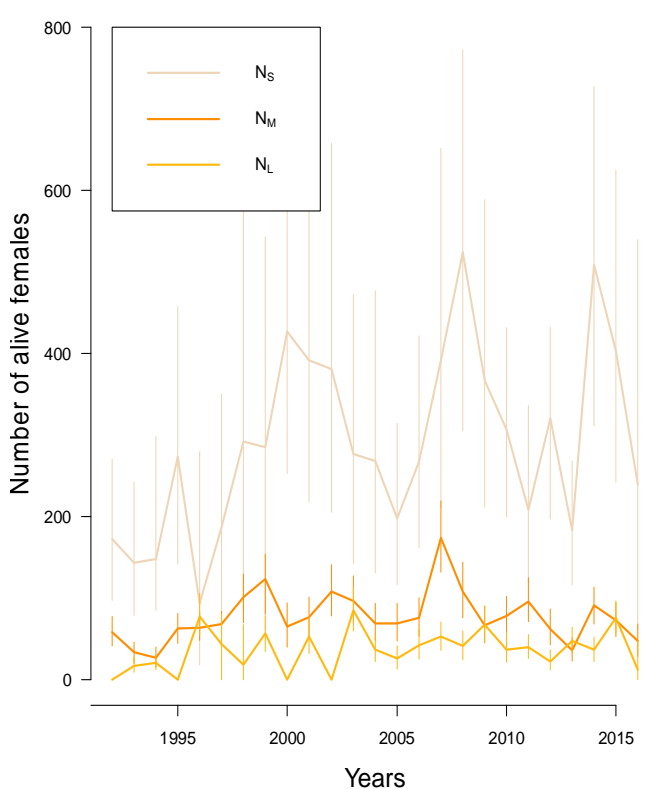

